\title{
Ambiente Digital para Ensino e Acompanhamento Personalizado de Estudantes com Autismo: proposta com Uso de Dispositivos Móveis
}

\author{
Martony Demes da Silva ${ }^{1}$, André Castelo Branco Soares ${ }^{1}$, Priscila Benitez ${ }^{2}$, \\ ${ }^{1}$ Universidade Federal do Piauí, PPGCC \\ Teresina - Piauí - Brasil \\ ${ }^{2}$ Universidade Federal do ABC, CMCC \\ Santo André - São Paulo - Brasil \\ mardemes@gmail.com,andre.soares@ufpi.edu.br,pribenitez@yahoo.com.br
}

Resumo. Estudantes com Transtorno do Espectro do Autismo (TEA) podem apresentar dificuldades relacionadas à aprendizagem acadêmica e para tal, são propostas estratégias diferenciadas, com uso de recursos tecnológicos, orientados por uma equipe profissional interdisciplinar. Este trabalho propõe uma estratégia de ensino com uso de uma aplicação para tablet. Como diferencial, o profissional pode elaborar as atividades com base no currículo da criança por meio de um software WEB. O ambiente digitalmTEA - foi avaliado em três estudantes com TEA e por uma profissional. Os resultados mostram que o ambiente permitiu a criação de atividades específicas para a demanda curricular de cada estudante com TEA, elaborado e aplicado por profissionais com conhecimentos básicos em computação, de maneira flexível e personalizada.

Abstract. Students with Autism Spectrum Disorder (ASD) may present difficulties related to academic learning, and for this purpose, differentiated strategies are proposed, using technological resources, guided by an interdisciplinary professional team. This work proposes a teaching strategy using a tablet application. As a differential, the professional can elaborate activities based on the child's curriculum through a WEB software. The digital environment - mTEA - was evaluated in three students with ASD and by a professional. The results show that the environment allowed the creation of specific activities for the curricular demand of each student with TEA, elaborated and applied by professionals with basic knowledge in computing, in a flexible and personalized way.

\section{Introdução}

Estudantes com Transtorno do Espectro do Autismo (TEA) apresentam déficits qualitativos na comunicação, assim como na interação social, além de interesses restritos e comportamentos estereotipados, de acordo com os manuais oficiais [APA, 2013]. Ademais, os estudantes apresentam variações na intensidade de manifestação dos sintomas, o que significa que alguns deles podem apresentar excessos comportamentais e outros déficits ou mesmo ausência de repertório comportamental.

Dentre as diferentes estratégias de trabalho apresentadas pela literatura, a Análise do Comportamento Aplicada (termo original em inglês, Applied Behavior Analysis) constitui-se em uma abordagem promissora para desenvolver o trabalho com essa população, devido aos conhecimentos comprovados cientificamente no tratamento de estudantes com TEA [Cooper et al. 2007]. 
Para desenvolver uma intervenção pautada nos princípios da ABA é necessário i) avaliar o desempenho do estudante, ii) elaborar atividades personalizadas para cada um deles, com base nos dados analisados em cada avaliação; iii) aplicar as atividades com registro dos dados; iv) analisar os dados de cada estudante; v) propor novas atividades a partir da análise dos dados de cada estudante [Cooper et al. 2007]. Para a implantação de um serviço em ABA com estudantes com TEA é necessário compor uma equipe interdisciplinar, que contemple diferentes áreas, com um mesmo objetivo educacional, a partir de uma mesma abordagem.

As atividades fundamentadas nos princípios da ABA podem ser aplicadas com papel e lápis (método tradicional) ou com uso de programa de computador (software). Contudo, para a elaboração das atividades, o profissional da equipe interdisciplinar deve ter domínio dos princípios básicos da $\mathrm{ABA}$, tais como, transformar os dados da avaliação em atividades de ensino, propor a estrutura do trabalho com base na tríplice contingência (estímulo antecedente, resposta e consequência), estabelecer critérios de aprendizagem, elaborar atividades que favoreçam a generalização, estabelecer uma sequência curricular das atividades, de modo que progridam de acordo com o ritmo individual de aprendizagem de cada estudante, dentre outras características [Cooper et al. 2007]. Quando tais atividades podem ser elaboradas em software ocorre a padronização da instrução, garantindo uma padronização na aplicação das atividades. Isso facilita que os pais possam aplicar as atividades em casa, assim como qualquer outro professional (por exemplo, professor em escolas regulares), além de otimizar a análise dos dados.

Além disso, as atividades realizadas com uso de software favorecem a disponibilização de dicas visuais, em contraste com as atividades desenvolvidas com uso de papel e lápis. As dicas visuais são consideradas estratégias fundamentais para a aprendizagem do estudante com TEA. Portanto, os softwares viabilizam o uso de cores, luzes, toques na tela, animações, automatizações, entre outros [Bernard-Opitz et al. 2004, Krantz and Mcclannahan 1998, Shabani et al. 2002].

Diferentes trabalhos foram desenvolvidos propondo software para ensino de estudantes com TEA [Chien et. Al. 2015], [Hiniker et. Al. 2013], [Escobedo et. Al. 2015], [Hani and Abu-Wandi et. Al. 2015], [Buzzi et. Al. 2012], [Passerino et. Al. 2010] e [Farias et. Al. 2014]. Entretanto, segundo nosso conhecimento, não existem soluções de software para tablets e smartphones com a capacidade de desenvolvimento de atividades personalizadas de acordo com as necessidades de cada estudante. A contribuição deste artigo é a proposta de um novo ambiente digital - $m T E A$ - que permite ao profissional educador elaborar de forma flexível (sem conhecimentos avançados de computação) atividades de ensino personalizadas, de acordo com as necessidades de cada estudante. $\mathrm{O}$ $m T E A$ viabiliza a elaboração, aplicação e análise dos resultados das atividades de ensino. Este artigo também apresenta uma avaliação do ambiente digital. O $m T E A$ foi utilizado por um profissional da área de psicologia como ferramenta para elaboração, aplicação e análise de resultados de atividades de ensino para 3 estudantes com TEA. Na avaliação, segundo o profissional, os estudantes se sentiram mais motivadas e apresentaram uma concentração maior comparado com o método tradicional.

As próximas seções deste artigo estão estruturadas da seguinte forma: a Seção 2 apresenta os trabalhos relacionados, a Seção 3 apresenta o $m T E A$, a Seção 4 detalha a metodologia usada para avaliação do $m T E A$, a Seção 5 discute os resultados e contribuições. A Seção 6 apresenta as considerações finais. 
VI Congresso Brasileiro de Informática na Educação (CBIE 2017)

Anais do XXVIII Simpósio Brasileiro de Informática na Educação (SBIE 2017)

\section{Trabalhos Relacionados}

O uso de software para incentivo ao desenvolvimento do estudante com autismo vem sendo bem-sucedido em pesquisas nos últimos anos [Aresti-Bartolome et al. 2014]. Recentemente, diferentes propostas de software foram desenvolvidas para auxiliar o ensino de estudantes com TEA. Dentre os softwares desenvolvidos para uso em desktop estão: Mestre, ProgLeit, GEIC, PELPS [Haydu, 2014] e ABCD SW [Buzzi et. Al. 2012]. Dentre esses, apenas o ABCD SW permite que o profissional educador crie novas atividades personalizadas as necessidades de cada estudante. Dentre os softwares voltados para uso em tablet estão: iCAN [Chien et. al. 2015], Go-Go-Games [Hiniker et. al. 2013], MOSOCO [Escobedo et. al. 2015], DISSERO [Hani and Abu-Wandi 2015], SCALA [Passerino et. al. 2010] e ABC Autismo [Farias et. al. 2014]. Os softwares desenvolvidos especificamente para tablets e smartphones são conhecidos também como Apps (sigla que vem do inglês Application). Este trabalho tem foco no uso de dispositivos móveis (tablets) e suas apps para auxílio de estudantes com TEA. Portanto, a seguir são detalhadas as soluções voltadas para uso em tablets.

O iCAN é um app para tablet baseado na prancha visual de comunicação PECS (Picture Exchange Communication System). O SCALA é um app para tablet que auxilia o estudante com TEA na comunicação alternativa baseado na prancha visual de comunicação PECS. O iCAN é utilizado para auxiliar a comunicação de crianças autistas não verbais. O Go-Go-Games apresenta um jogo para tablet utilizado como terapia para apoiar educação de crianças autistas. O MOSOCO é um App para dispositivos móveis que auxilia as crianças com autismo a praticar habilidades sociais em situações reais. Já o DISSERO é também um app que visa melhorar cognição das crianças e suas habilidades sociais aumentando o processo de aprendizagem. O ABC Autismo é app para tablet que possui atividades (de diferentes níveis) que auxiliam no processo de alfabetização de estudantes com autismo. ABCD SW é um software WEB concebido para uso em desktop, no qual o tutor cria atividades com base no desempenho do estudante. Seu foco é auxiliar o processo de aprendizagem de estudantes com TEA. Por ser um software WEB, o ABCD SW pode ser acessado por meio do navegador WEB do tablet. Entretanto, por não ser uma aplicação nativa do tablet, o ABCD SW não oferece a precisão de toques na tela touch, conforme é observado nos apps desenvolvidos especificamente para tablet.

Nenhum dos trabalhos relacionados reúne em uma mesma solução as seguintes características: i) Elaboração de atividades pelo profissional educador de acordo com o currículo e a necessidade de cada estudante com TEA, sem requer conhecimentos avançados em computação, ii) realização das atividades com uso de tablet explorando as funcionalidades da tela touch; iii) acompanhamento da evolução do desempenho de cada estudante com TEA. Portanto, este artigo propõe o ambiente digital $m T E A$ que reúne todas as características citadas acima.

\section{MTEA}

O mTEA é composto por três softwares: i) servidor que armazena os dados na nuvem (mTEA-Server), ii) software WEB (mTEA-WEB) usado para elaboração das atividades personalizadas e iii) app para tablet/smartphone usado para aplicação das atividades de ensino aos estudantes com TEA (mTEA-App).

O $m T E A-W E B$ foi desenvolvido com linguagem de programação Java (Java 8). Utilizou-se também as tecnologias Java Server Faces (JSF) e Primefaces, que permitiram 
VI Congresso Brasileiro de Informática na Educação (CBIE 2017)

Anais do XXVIII Simpósio Brasileiro de Informática na Educação (SBIE 2017)

acelerar o processo de programação visto que estes frameworks dispões componentes de softwares prontos para utilização em interfaces com usuário. O mTEA-Server é um servidor composto por um serviço de aplicação, o Wildfly, um banco de dados Postgres. O padrão de projeto utilizado foi agregação de MVC (Model View Controller) e DAO (Data Access Object). O $m$ TEA-App foi desenvolvido para a plataforma Android. Com o tablet, a criança usa o $m T E A-A p p$ que funciona com um player das atividades elaboradas pelo profissional educador. O mTEA-App está disponível para instalação no link: https://goo.gl/xoLL2u. O acesso ao mTEA-WEB está disponível em: http://bit.ly/2t76rs0. Usuário: cbie e senha:cbiel.

Para viabilizar a elaboração de atividades personalizadas pelo profissional educador foi utilizado o conceito de template. Template é a estrutura que define o tipo da atividade de ensino, especificando quais os parâmetros podem ser alterados pelo profissional educador. A Figura 1 apresenta a relação entre o template e a atividade. Além disso, destaca o que pode ser desenvolvido pelo profissional especialista em ABA e o que é desenvolvido pela equipe de programadores.

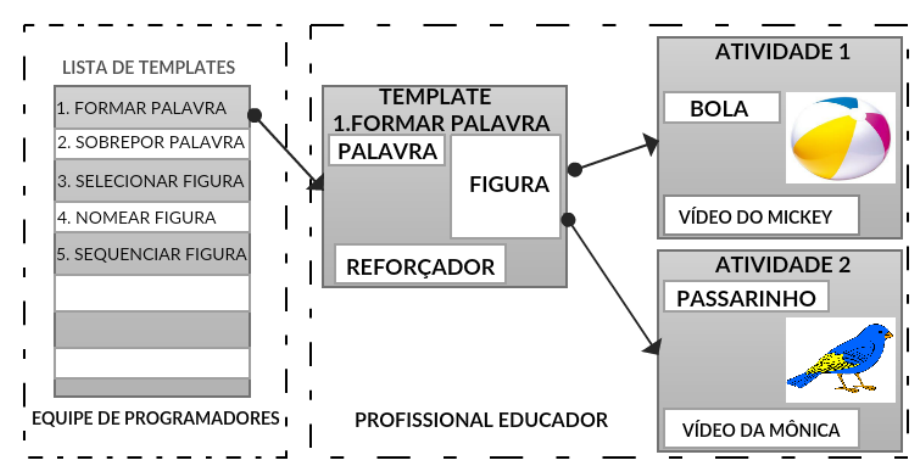

Figura 1. Principais componentes do mTEA.

A Figura 1 destaca à esquerda, a lista de templates que é desenvolvida previamente pelos programados de software. À direita da Figura 1, é ilustrado o uso desses templates pelo profissional educador. Por exemplo, no uso do template formar palavra (Figura 1), o educador usa o mTEA-WEB para criar a ATIVIDADE 1, formar a palavra BOLA. Além disso, para mesma ATIVIDADE 1, o educador define uma ilustração como dica visual (ilustração da BOLA). O educador também adiciona um vídeo que será apresentado ao final da atividade, servindo como reforçador. $\mathrm{O}$ educador deve escolher o vídeo de acordo com a preferência do estudante, para estimular a realização das atividades propostas. Observa-se ainda na Figura 1 a elaboração da ATIVIDADE 2, onde o educador elabora outra atividade (formar palavra PASSARINHO) com o mesmo template formar palavra. Vale destacar que a escolha dos parâmetros de cada template permite a elaboração de atividades personalizadas, de acordo com o currículo de cada estudante com TEA.

A Tabela 1 descreve os cinco templates disponíveis no mTEA.

Tabela 1. Descrição dos cinco templates existentes no mTEA

\begin{tabular}{|l|l|}
\hline Template & Descrição \\
\hline Formar palavras & Formar a palavra correspondente, arrastando cada letra para espaço em branco \\
\hline Sobrepor palavras: & Arrastar cada letra sobre a letra correta na palavra acima \\
\hline Selecionar Figuras & Selecionar figuras idênticas (memória) \\
\hline Nomear figuras: & Nomear as figuras que surgirem \\
\hline Sequenciar figuras & Selecionar figura, seguindo a sequência que surgir para criança \\
\hline
\end{tabular}


VI Congresso Brasileiro de Informática na Educação (CBIE 2017)

Anais do XXVIII Simpósio Brasileiro de Informática na Educação (SBIE 2017)

\section{Metodologia de avaliação}

O presente trabalho foi aprovado pelo Comite de Etica em Pesquisas em Seres Humanos da Universidade Federal de São Carlos (CAAE 60273816.4.0000.5504) e as atividades iniciaram após aprovação e assinatura dos termos de consentimento livre e esclarecido pelos participantes

\subsection{Participantes}

Para a realização do presente estudo foi selecionado um profissional e três estudantes com TEA. Os critérios para seleção do profissional foram: a) desenvolver um trabalho profissional com estudantes com TEA, b) ter conhecimento básico de informática, c) compor uma equipe interdisciplinar com abordagem teórica em ABA. Para os estudantes, os critérios foram: a) ser atendido pelo profissional participante do estudo, b) apresentar diagnóstico médico de TEA.

A profissional-participante do estudo tinha formação na área da Psicologia e da Pedagogia e atuava há oito anos na área da educação especial, com intervenções envolvendo estudantes com autismo e ABA. Os estudantes com TEA, denominados como E1, E2 e E3, tinham quatro, cinco e oito anos, respectivamente e eram atendidos pela profissional há mais de um ano cada um deles. E1 e E3 eram meninos e E2 menina.

\subsection{Situação e Materiais}

A elaboração e aplicação das atividades pela profissional-participante ocorreram em um centro especializado de atendimento, que conta com uma equipe interdisciplinar e estudantes com TEA e outras deficiências e transtornos. Como material foi utilizado um Tablet Samsung Galaxy Tab A 16GB de memória, 8 polegadas com internet via 4G ou Wi-Fi, plataforma Android e Processador Quad Core.

\subsection{Variáveis}

A pesquisa de cunho qualitativo foi realizada com base no relato de um profissionalparticipante que atuou com três estudantes com TEA. E para tal, foram definidas as variáveis do estudo. A variável independente do estudo se referiu a construção do $m T E A$ para a proposição das atividades de ensino pelo profissional-participante do estudo. As variáveis dependentes mensuradas qualitativamente ao longo do estudo se referiram ao desempenho do profissional-participante na elaboração e aplicação das atividades, mensurado por um questionário elaborado para essa finalidade; e, adicionalmente, ao desempenho dos estudantes com TEA nas atividades propostas pela profissionalparticipante.

\subsection{Procedimento}

Inicialmente, o ambiente $m T E A$ foi desenvolvimento pelos pesquisadores, conforme descrito na seção anterior. Em seguida, foi elaborado e aplicado as atividades pelo profissional-participante do estudo.

A profissional-participante do estudo recebeu orientações sobre como utilizar o $m T E A$, dadas pelos pesquisadores. Conforme a profissional-participante apresentava dúvidas era feito o contato direto com os pesquisadores para esclarecimentos e fornecimento de feedbacks. Esse conjunto de orientações estão sendo sistematizados para posterior confecção de um manual sobre o uso do ambiente. Dessa maneira a profissionalparticipante teve acesso ao mTEA-WEB para cadastrar os estudantes (E1, E2 e E3). Em 
VI Congresso Brasileiro de Informática na Educação (CBIE 2017)

Anais do XXVIII Simpósio Brasileiro de Informática na Educação (SBIE 2017)

seguida, a profissional-participante elaborou as atividades que cada um deles, de acordo com as necessidades individuais que apresentavam. Para o cadastro das atividades utilizou-se a base de templates disponíveis no m-TEA. A Figura 2 relaciona a visualização do educador ( $m T E A-W E B$ ) na elaboração da atividade formar palavra BOLA, como a visualização do estudante ( $m T E A-A p p)$ na realização a respectiva atividade.

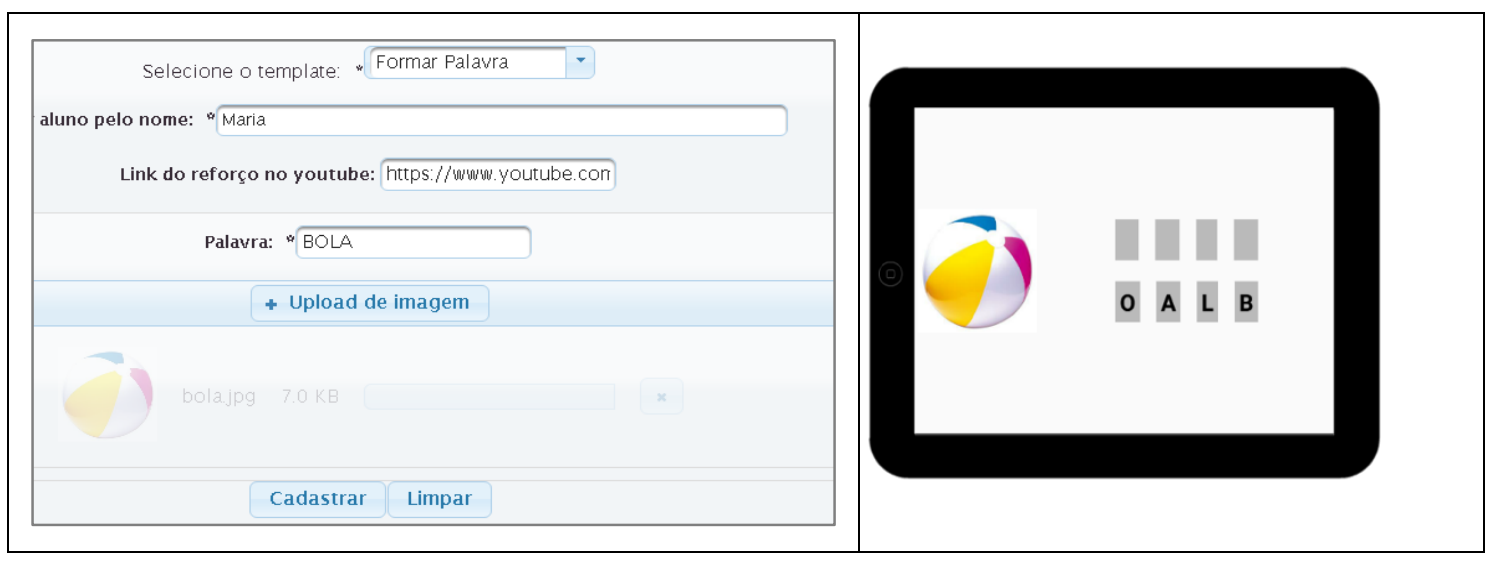

Figura 2. Telas do $m$ TEA.

Em seguida, a profissional-participante aplicou as atividades com os estudantes em usando o m-TEA-App no tablet. A Figura 2, lado direito, ilustra o design da atividade de formar palavra no mTEA-App visualizado pelo estudante. Destaca-se que a profissional utilizou uma imagem como dica visual.

E, por último, a profissional respondeu ao questionário composto por sete perguntas que versavam sobre a avaliação do mTEA. No geral, buscou-se comparar o mTEA aos métodos tradicionais, destacando os pontos negativos e positivos do ambiente, além da motivação e da concentração dos estudantes para a realização das atividades, a viabilidade para uso do mTEA em situação domiciliar, aplicado pelos pais e a qualidade das análises de dados propostas pelo mTEA.

Tabela 2. Questões para a profissional-participante responder.

\begin{tabular}{|c|l|}
\hline $\mathrm{N}$ & Descrição da questão \\
\hline 1 & $\begin{array}{l}\text { O ambiente digital proposto, considerando as atividades aplicadas, é equivalente aos métodos tradicionais? Por } \\
\text { que? }\end{array}$ \\
\hline 2 & Pontos negativos do ambiente digital em comparação com o método tradicional? \\
\hline 3 & $\begin{array}{l}\text { Pontos positivos do ambiente digital em comparação com o método tradicional? As questões } 4 \text { e } 5 \text { se referem } \\
\text { ao desempenho dos estudantes com TEA durante a aplicação das atividades no ambiente digital: }\end{array}$ \\
\hline 4 & Os estudantes ficaram mais ou menos motivadas para realização das atividades? \\
\hline 5 & $\begin{array}{l}\text { Qual o nível de concentração das crianças ao usar o ambiente digital? É possível fazer uma comparação do } \\
\text { nível de atenção das crianças em relação ao método tradicional? }\end{array}$ \\
\hline 6 & $\begin{array}{l}\text { Em sua opinião, o ambiente é útil para realização de tarefas na casa das crianças com acompanhamento dos } \\
\text { pais? }\end{array}$ \\
\hline 7 & $\begin{array}{l}\text { Os dados dispostos nos relatórios e gráficos do ambiente web possibilitam realizar um bom acompanhamento } \\
\text { do rendimento da criança? }\end{array}$ \\
\hline
\end{tabular}

Os dados foram analisados de acordo com o questionário respondido pela profissional-participante, assim como pelos desempenhos dos três estudantes com TEA, nas diferentes atividades propostas pela profissional-participante. E, de maneira geral, foram analisadas as propostas de melhorias e encaminhamentos futuros para posterior aprimoramento do $m T E A$. 
VI Congresso Brasileiro de Informática na Educação (CBIE 2017)

Anais do XXVIII Simpósio Brasileiro de Informática na Educação (SBIE 2017)

\section{Resultados e Contribuições}

Os resultados foram agrupados em duas categorias que se referem ao desempenho dos estudantes nas atividades realizadas pelo $m T E A$ e elaboradas pela profissionalparticipante do estudo, assim como em relação à avaliação dada pela profissionalparticipante ao ambiente. A Figura 3 mostra os desempenhos dos estudantes nas atividades. E1 realizou quatro atividades, de acordo com o seu currículo personalizado: sobreposição de palavras, formação de palavras - PAPAI, identificação de figuras similares e sequência numérica. E2 realizou cinco atividades, com exceção da sequência numérica. E3 realizou cinco atividades, com exceção da nomeação de figuras.

O percentual de acertos apresentado na Figura 3 mostra o desempenho de cada um deles nas diferentes atividades. Cada estudante realizou quatro vezes a mesma atividade e assim, foi realizado o cálculo de cada desempenho, registrado no mTEA. Por exemplo, A2 realizou quatro vezes a atividade de sobreposição de palavras e respondeu corretamente todas as tentativas, o que resultou em 100\% de acertos. Já na atividade de formar a palavra PAPAI, respondeu incorretamente nas duas primeiras tentativas e corretamente nas duas últimas, o que resultou em $50 \%$ de acertos.

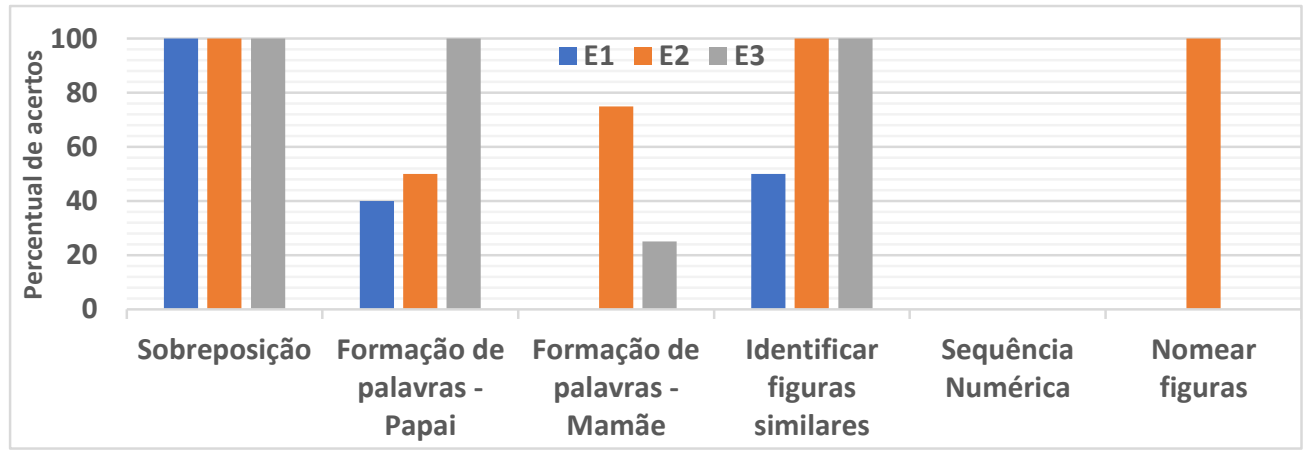

Figura 3. Desempenho dos três estudantes com TEA nas atividades avaliadas no mTEA. A coluna azul se refere ao desempenho de E1, a coluna laranja ao desempenho de E2 e cinza E3.

A Tabela 3 mostra as respostas da profissional-participante para as perguntas do questionário aplicado.

Tabela 3. Categorias de análise das respostas da profissional-participante, em relação à avaliação ao mTEA.

\begin{tabular}{|c|c|c|}
\hline \multicolumn{2}{|c|}{ Categorias } & Respostas \\
\hline 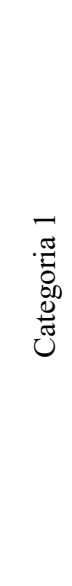 & $\begin{array}{l}\text { Avaliação do } \\
\text { ambiente digital em } \\
\text { comparação às } \\
\text { atividades aplicadas } \\
\text { manualmente } \\
\text { (Questões } 1,2 \text { e 3) }\end{array}$ & $\begin{array}{l}\text { "O ambiente facilita a aplicação das atividades com os estudantes com TEA, } \\
\text { devido aos recursos que são possíveis de serem utilizados com o uso da tecnologia, } \\
\text { além de favorecer uma análise de dados mais rápida, em comparação ao trabalho } \\
\text { manual". } \\
\text { "O ambiente apresenta uma interface amigável e intuitiva, sem necessidade de } \\
\text { conhecimento prévio na área da computação, além da facilidade para aplicação no } \\
\text { tablet ou mesmo em smartphone. Outro ponto se refere a possibilidade de elaborar } \\
\text { atividades, de acordo com o desempenho do estudante e adaptá-las, conforme sua } \\
\text { necessidade. O registro dos desempenhos automático pelo ambiente economiza o } \\
\text { tempo de aplicação do profissional". } \\
\text { "Como em qualquer programa, podem ser realizadas melhorias, como, modificar } \\
\text { a consequência reforçadora, de acordo com os interesses do estudante, por } \\
\text { exemplo, ao finalizar a atividade, acesso automático à um vídeo no youtube; } \\
\text { fornecer instruções orais para serem dadas diretamente pelo ambiente, conforme } \\
\text { cada atividade e especificar as figuras/palavras no relatório de cada atividade". }\end{array}$ \\
\hline
\end{tabular}


VI Congresso Brasileiro de Informática na Educação (CBIE 2017)

Anais do XXVIII Simpósio Brasileiro de Informática na Educação (SBIE 2017)

\begin{tabular}{|c|c|c|}
\hline 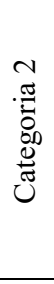 & $\begin{array}{l}\text { Desempenho dos } \\
\text { estudantes com TEA } \\
\text { nas atividades } \\
\text { (Questões } 4 \text { e 5) }\end{array}$ & $\begin{array}{l}\text { "Ao visualizar as atividades no tablet, eles sorriam e solicitavam para faze-las, o } \\
\text { que significa que estavam motivados para realização de tais atividades. É } \\
\text { importante destacar que tal comportamento não ocorria com as atividades no } \\
\text { papel". } \\
\text { "Os estudantes estabeleciam contato visual permanente com os estímulos presentes } \\
\text { nas atividades, o que favoreceu maior número de acertos em cada uma delas, o que } \\
\text { significa que eles estavam mais concentrados". }\end{array}$ \\
\hline 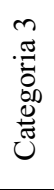 & $\begin{array}{l}\text { Uso do ambiente pelos } \\
\text { pais (Questão 6) }\end{array}$ & $\begin{array}{l}\text { “A aplicação das atividades é muito fácil e pode ser utilizada por qualquer } \\
\text { populaça, inclusive pelos pais. Devido a automatização das atividades, o } \\
\text { profissional pode programar uma sequência e deixar disponível para que os pais } \\
\text { realizem com seus filhos, de modo a ampliar o tempo de intervenção, nas } \\
\text { respectivas residências". }\end{array}$ \\
\hline 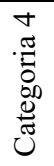 & $\begin{array}{l}\text { Análise de dados } \\
\text { oferecida } \quad \text { pelo } \\
\text { ambiente (Questão 7) }\end{array}$ & $\begin{array}{l}\text { "Os relatórios emitidos pelo ambiente otimizam a análise de dados, por agilizarem } \\
\text { o cálculo de acertos e erros, facilitando assim as tomadas de decisão sobre o } \\
\text { currículo de cada estudante com TEA, contudo, precisam ser mais específicos, em } \\
\text { relação ao desempenho para cada palavra e/ou figura". }\end{array}$ \\
\hline
\end{tabular}

Dentre as dúvidas registradas pela profissional-participante destacam-se: a) particularidades de cada atividade (por exemplo, sobre a necessidade de fazer upload da mesma figura duas vezes para a atividade de identificação de figuras similares - jogo da memória), b) questões técnicas (por exemplo, o link digitado para acesso ao vídeo no youtube após realização da atividade não funcionou; ao criar uma atividade poder aproveitar atividade de outros estudantes, de modo a economizar o tempo de elaboração das atividades pelo profissional; estabelecer critério de repetição da atividade; fornecer instruções orais pelo próprio ambiente).

\section{Discussão e Considerações Finais}

A presente pesquisa objetivou elaborar e avaliar o ambiente $m T E A$ para o ensino personalizado de habilidades básicas para estudantes com TEA, a partir da elaboração e da aplicação das atividades por um profissional que trabalhava com estudantes com TEA. É fundamental ressaltar que tais estudantes podem apresentar dificuldades relacionadas às diferentes áreas do desenvolvimento, a destacar: linguagem, desenvolvimento motor, autocuidados, habilidades sociais e cognição, especialmente no que concerne à aprendizagem acadêmica, no tocante as habilidades de leitura e escrita.

O ambiente proposto foi viabilizado pela integração de um sistema WEB (mTEAWEB) e do aplicativo para dispositivo móvel (smartphone e tablet). O mTEA-WEB possibilitou a elaboração de atividades personalizadas pela profissional que integra uma equipe interdisciplinar que trabalhava com estudantes com TEA, além de permitir o acompanhamento das atividades e a análise do desempenho individual de cada um deles, o que contribui para a programação de um currículo de Ensino, de acordo com os princípios da ABA (Cooper et al., 2007).

Neste sentido, a principal inovação com mTEA foi permitir que o profissional da equipe multidisciplinar elaborasse as atividades individualizadas, com base no currículo personalizado de cada estudante, além do registro automático do desempenho dos estudantes com TEA, em conformidade com a avaliação da profissional-participante na Categoria 1 da Tabela 3. Outra característica importante deste ambiente se refere a possibilidade dos pais realizarem as atividades com os seus filhos, em suas residências, de acordo com o relato da profissional-participante na Categoria 3 da Tabela 3. Assim, os responsáveis pelas crianças podem controlar o tempo de realização das atividades e acompanhar o rendimento dos seus filhos. Ademais, o relatório fornecido pelo ambiente 
otimizou a análise de dados e a aplicação das atividades, visto que nas atividades manuais, o profissional necessita aplicar e registrar manualmente o desempenho do estudante com TEA, em cada atividade. Já no mTEA, o profissional não necessita registrar os dados, pois tais registros são realizados automaticamente, segundo o relato da profissionalparticipante na Categoria 4 da Tabela 3 . Com relação à motivação dos estudantes com TEA para realização das atividades no $m T E A$ foi considerada como positiva, de acordo com os relatos da profissional-participante para a Categoria 2 da Tabela 3, o que corrobora os desempenhos apresentados na Figura 3, uma vez que realizaram todas as atividades propostas em seus currículos personalizados.

As dicas visuais fornecidas nas atividades de formação e sobreposição das palavras e de sequência numérica favoreceram o maior desempenho dos estudantes nas atividades, o que corrobora os achados descritos na literatura (BERNARD-OPITZ; ROSS, 2004; KRANTZ; MCCLANNAHAN, 1998; SHABANI; KATZ; WILDER; BEAUCHAMP, 2002). O conjunto de dados apresentados identificam os avanços do $m T E A$, em comparação aos ambientes com domínio público disponíveis para a elaboração de atividades por profissionais. Além disso, são propostas sugestões para aprimoramento do programa em versões futuras, no que dizem respeito aos seguintes aperfeiçoamentos: a) após o término da atividade ter acesso automático ao vídeo do youtube, de acordo com a preferência de cada estudante com TEA; b) copiar a atividade para diferentes estudantes, de modo a otimizar a elaboração; c) estabelecer critério de repetição da atividade; d) fornecer instruções orais pelo próprio ambiente; e) no relatório dos dados, apresentar o desempenho em cada atividade de acordo com cada figura e cada palavra trabalhada e, ainda, descrever a resposta do estudantes, ao invés de apenas quantificá-la; f) ampliar o número de templates para outras atividades.

Os dados do presente estudo alcançaram os objetivos propostos em relação a elaboração e avaliação do $m T E A$, com dados fundamentais para continuidade e aprimoramento do ambiente, visando a disseminação e uso por diferentes agentes educacionais. Os resultados mostram que o mTEA permitiu a criação de atividades específicas para a demanda curricular de cada estudante com TEA, elaborado e aplicado por profissionais leigos na área computacional, o que sugere que o ambiente apresenta uma interface amigável, além de favorecer o ensino das habilidades básicas supracitadas.

Para trabalhos futuro, pretende-se realizar novos experimentos envolvendo maior número de profissionais da equipe multidisciplinar, assim como com maior número de estudantes com TEA, além da elaboração de outros templates de atividades visando atingir um maior número de habilidades de ensino e um manual com explicações técnicas para que pessoas leigas possam utilizar o $I T E A$ em diferentes localidades.

\section{Referências}

APA. American Psychiatric Association. Diagnostic and statistical manual of mental disorders. 5th ed. Arlington, VA: American Psychiatric Publishing, 2013.

Aresti B., Nuria and Garcia Zapirain, Begonya. Technologies as Support Tools for Persons with Autistic Spectrum Disorder: A Systematic Review. International Journal of Environmental Research and Public Health, v.11, p. 7767-7802, 2014.

Bernard-Opitz, V., Ross, K. and Nakahoda-Sapuan, S. Enhancing social problem solving in children with autism and normal children with computer assisted instruction. Journal of Autism and Developmental Disorder, v. 31, n. 4, p. 377-384, 2004. 
VI Congresso Brasileiro de Informática na Educação (CBIE 2017)

Anais do XXVIII Simpósio Brasileiro de Informática na Educação (SBIE 2017)

Buzzi, M. Claudia, Buzzi, M., Gazzé, D., Senette, C. and Tesconi, M. ABCD SW: autistic behavior \& computer-based didactic software. W4A, ACM (2012), 282.

Chien, M., Jheng, C., Lin, N., Tang, H., Taele, P., Tseng, W. and Chen, Mike. iCAN: A tablet-based pedagogical system for improving communication skills of children with autism. Int. J. Human-Computer Studies, v.73, p. 79-80, 2015

Cooper, J. O., Heron, T. E. and Heward, W. L. Applied behavior analysis. (2a ed.). Upper Saddle River, NJ: Pearson Education, 2007.

Escobedo L., Nguyen D. H., Boyd L. A., Hirano Sen H., Rangel A. , Garcia-Rosas D., Tentori D., Hayes G. R. MOSOCO: A Mobile Assistive Tool to Support Children with Autism Practicing Social Skills in Real-Life Situations. In Proceedings of the SIGCHI Conference on Human Factors in Computing Systems, p. 2589-2598, 2015.

Farias, E., B., Silva, Leandro W. C. and Cunha, M. X. C. ABC AUTISMO: Um aplicativo móvel para auxiliar na alfabetização de crianças com autismo baseado no Programa TEACCH. Anais do Simpósio Brasileiro de Sistema de Informação, 2014.

Hani, H. and Abu-Wandi, R. DISSERO Mobile Application for AUTISTIC Children's. Proceedings of the International Conference on Intelligent Information Processing, Security and Advanced Communication, s. IPAC '15, p. 90:1--90:6, 2015.

Haydu, V. B. O modelo da equivalência de estímulos na forma de jogos educativos para o ensino leitura e escrita em contexto coletivo. 2014. In: Haydu, V. B., Fornazari, S. A. and Estanislau, C. R. (Orgs.). Psicologia e Análise do Comportamento: Conceituações e Aplicações à Educação, Organizações, Saúde e Clínica. 1ed. Londrina: UEL, 2014, v. 1, p. 177-197.

Hiniker A., Wong, D. J. and Williamson H. Go go games: therapeutic video games for children with autism spectrum disorders, Proceedings of the 12th International Conference on Interaction Design and Children, p.463-466, June 24-27, 2013.

Krantz, P. J. and Mcclannahan, L. E. Social interaction skills for children with autism: A script-fading procedure for beginning readers. Journal of Applied Behavior Analysis, v. 31, p. 191-202, 1998.

Passerino, L. M., AVILA, B. G. and BEZ, M. R. SCALA: um Sistema de Comunicação Alternativa para o Letramento de Pessoas com Autismo. RENOTE. Revista Novas Tecnologias na Educação, v.1, p. 1-10, 2010.

Sahabani, D. B.; Katz, R. C.; Wilder, D. A.; Beauchamp, K. and Taylor, C. R.; FISCHER, K., J. Increasing social initiations in children with autism: Effects of a tactile prompt. Journal of Applied Behavior Analysis, v. 35, p. 79-83, 2002. 